\title{
Can Laterally Overgrown GaN Layers be free of Structural Defects?
}

\author{
D. Cherns and Z. Liliental-Weber*
}

H.H. Wills Physics Laboratory, University of Bristol, Tyndall Avenue, Bristol BS8 1TL, UK *permanent address: Lawrence Berkeley Laboratory, Materials Science Division, Berkeley, CA 94720, USA

\begin{abstract}
Transmission electron microscopy has been used to examine dislocations present in an epitaxial laterally overgrown (ELOG) sample of GaN grown on (0001)sapphire. Studies of both plan-view and cross-sectional samples revealed arrays of dislocations present in the (11-20) boundary between the seed and the wing (overgrown) material and at the meeting front between adjacent wings, as well as dislocations in the form of half-loops extending into the wing regions. Both the boundary and half-loop dislocations had $1 / 3<11-20>$ Burgers vectors which were either perpendicular (boundary dislocations) or at $30^{\circ}$ (half-loops) to the boundary plane. Large angle convergent beam electron diffraction was used to show that the boundary dislocations and halfloops correlated respectively with tilts and twists of the wing material about (11-20). A model is proposed whereby the half-loops are generated from threading dislocations by shear stresses acting along the stripe direction. The origin, and elimination, of these stresses is discussed.
\end{abstract}

\section{Introduction}

The epitaxial lateral overgrowth (ELOG) method was introduced in order to reduce the density of threading dislocations generated in $(0001) \mathrm{GaN}$ films grown on highly latticemismatched substrates such as (0001)sapphire. In the ELOG method, a mask, such as $\mathrm{SiO}_{2}$, is deposited on a part-grown $\mathrm{GaN}$ layer. On resuming the growth, $\mathrm{GaN}$ does not nucleate on the mask material. Growth then proceeds laterally from seed columns of $\mathrm{GaN}$ which grow through gaps etched into the mask. Eventually, the GaN "wings" meet to produce a continuous film. Provided threading dislocations continue to propagate in the seed material close to the c-axis as in conventional $\mathrm{GaN}$ growth, the wing regions should be substantially dislocation-free.

It is now clear that this simple model of ELOG growth does hold entirely, with significant, if lower, densities of dislocations reported for the wing material. Estimates vary widely, from, for example, below $10^{6} \mathrm{~cm}^{-2}$ [1] to nearly $10^{8} \mathrm{~cm}^{-2}$ [2], with dislocation densities in the seed material in the range $10^{9}-10^{10} \mathrm{~cm}^{-2}$. In addition, some observations show significant bending of the wings, which may lead to the generation of new defects [3]. Such behaviour may result from the high stresses generated by thermal mismatch between the $\mathrm{GaN}$ and the mask material [4].

In this paper, we use transmission electron microscopy (TEM) and large angle convergent beam electron diffraction (LACBED) to correlate the presence of dislocations in the wing regions of an ELOG sample with measurements of the local strain. A new mechanism of dislocation propagation in the wing material is proposed which is driven by shear stresses and involves both glide and climb. The implications of the results for reducing dislocation densities are considered. 


\section{Experimental}

The sample studied was grown by metal-organic chemical vapour deposition on (0001) sapphire. After $2.75 \mu \mathrm{m}$ of $\mathrm{GaN}$ had been grown, a $0.75 \mu \mathrm{m}$ thick $\mathrm{SiO}_{2}$ layer was deposited and etched to leave trapezoidal stripes of $\mathrm{SiO}_{2}$ with $11 \mu \mathrm{m}$ spacing and $3.5 \mu \mathrm{m}$ windows, and aligned along the [1-100] direction. After the mask preparation, a further $5 \mu \mathrm{m}$ of GaN was grown such that the wings were fully coalesced.

TEM studies were carried out on both plan-view and cross-sectional samples prepared by standard methods of mechanical polishing, dimpling and ion-thinning to perforation. Studies were carried out in a Philips EM430 microscope operating at 250kV. Fig. 1 shows micrographs of a cross-sectional sample showing the seed material on the right hand side, and part of an adjacent wing. In the bottom left hand corner of the micrographs, the edge of the $\mathrm{SiO}_{2}$ can be seen. The micrographs have been taken in two-beam imaging conditions with the g-vectors arrowed. In addition to threading dislocations in the seed material which lie approximately along the c-axis (vertical), there is an array of dislocations which appear as a boundary between the seed and wing regions. In addition, at roughly $0.5 \mu \mathrm{m}$ intervals along the c-axis, there are sets of dislocations which extend well into the wing region. These dislocations, as well as the boundary dislocations, are in strong contrast in fig. 1(a), but show residual contrast in fig. 1(b) consistent with $\mathbf{g . b}=0$ where $\mathbf{b}$ is the Burgers vector. This implies that the Burgers vectors must be of $1 / 3<11-20>$, or a-, type. Moreover, the straight appearance of both the wing and the boundary dislocations in fig. 1(b), where basal plane is nearly edge-on, confirms that the dislocations lie in the basal plane.

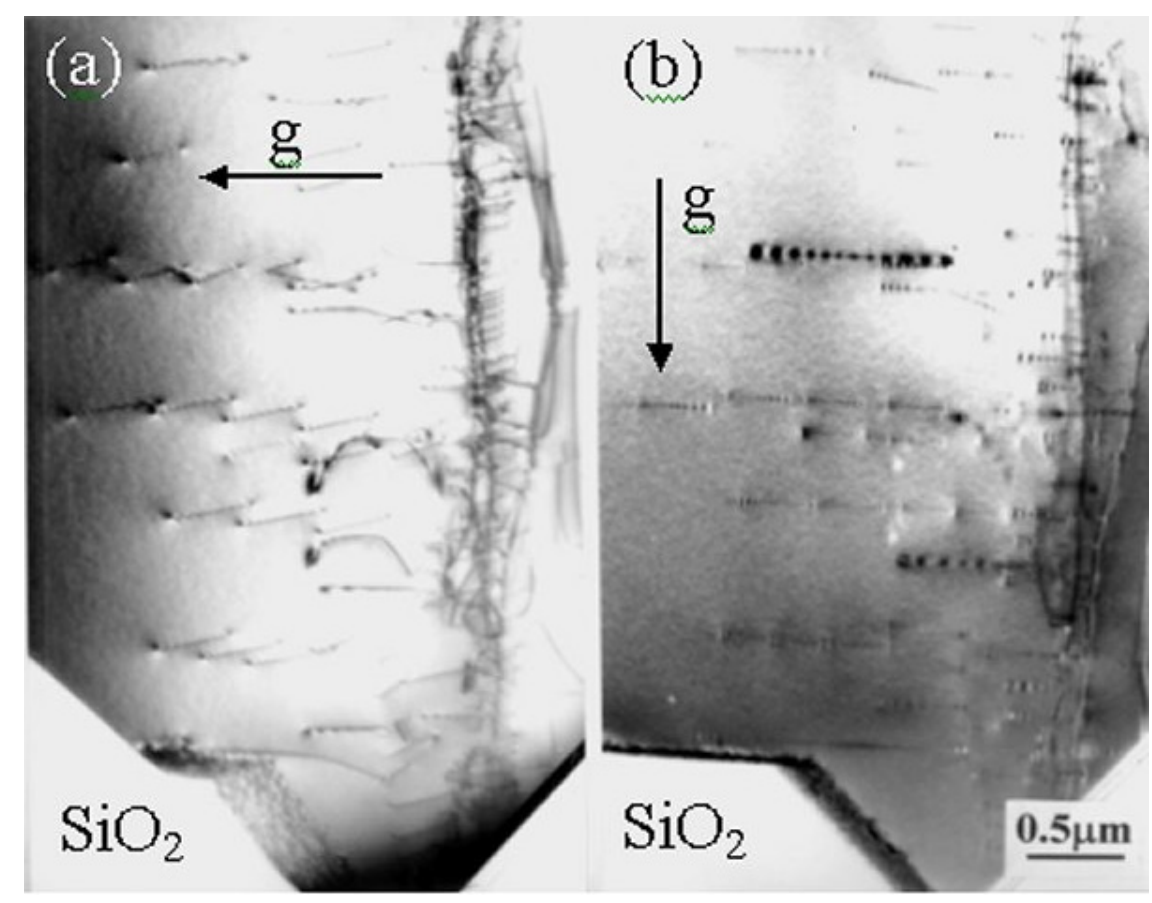

Fig. 1. Bright field images from a cross-sectional sample taken in two-beam conditions with (a) $g=01-10$ and (b) $g=000-2$, showing a region around a seed/wing boundary. In (b), the dislocations in the wing and in the seed/wing boundary show a residual contrast with strong depth oscillations. 


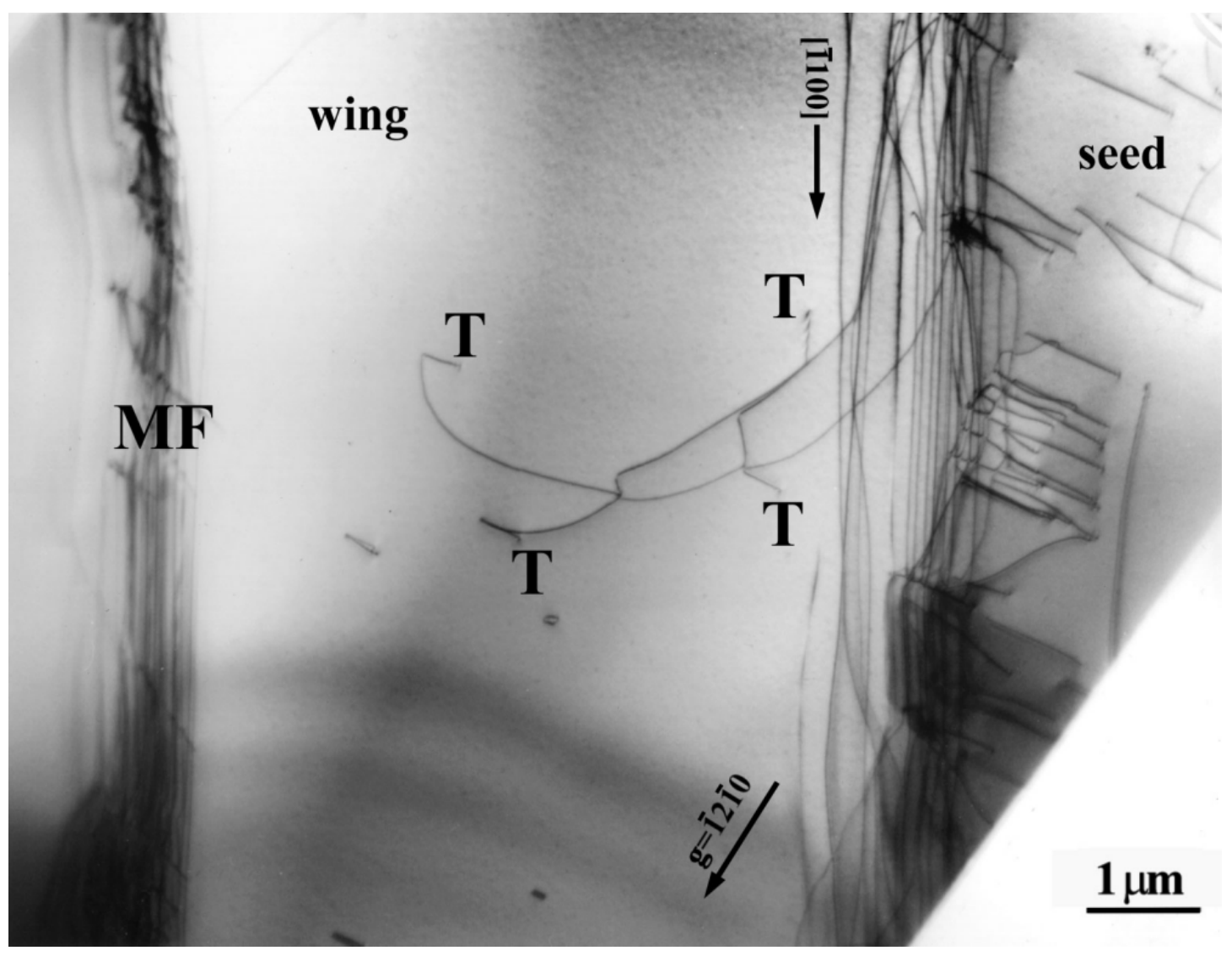

Fig. 2. Two-beam bright field image from a plan-view sample showing sets of dislocations at the seed/wing and wing/wing boundaries and two half-loop dislocations extending into the wing region. The half-loop dislocations end in threading segments $T$.

Fig. 2 shows a two-beam image from a plan-view sample which was prepared by backthinning towards the growth surface. Although only the top $0.5 \mu \mathrm{m}$ or so of deposit remains, we see essentially the same features visible in fig. 1. The sample has been tilted so that threading dislocations in the seed material are seen in projection (right hand side). In addition arrays of long straight dislocations delineate boundaries between the seed and wing regions and between adjacent wings (left hand side). Curved dislocations, or half-loops, are also apparent in the wing regions. Their contrast, confirmed by tilting experiments, showed that they lie predominantly in the basal plane. At the ends of the half-loops situated in the wing region, there are threading segments which go towards the substrate side of the film.

More detailed studies of the half-loops showed that they were present in most wing regions and were usually linked to either to the seed/wing or to the wing/wing boundary. Fig. 3 shows a series of such dislocations emanating from both sides of a wing/wing boundary. A Burgers vector analysis showed that the half-loops had Burgers vectors of 1/3[1-210] (dislocations A) or 1/3[2-1-10] (dislocations B) where the Burgers vectors (arrowed) are at angles of $30^{\circ}$ to the stripe direction. Most of the boundary dislocations had Burgers vectors $1 / 3$ [11-20], i.e. perpendicular to the stripe direction. 


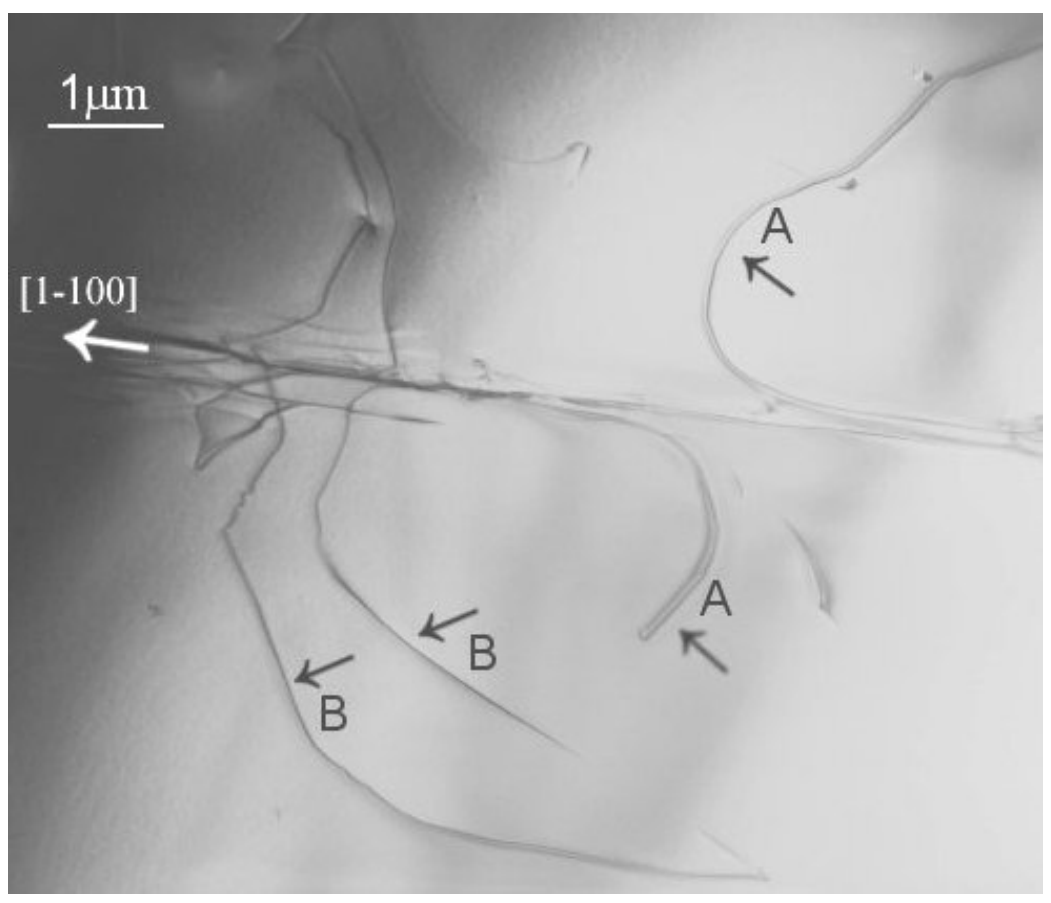

Fig. 3. Bright field micrograph from a plan-view sample showing a series of half-loop dislocations $A$ and $B$ on either side of a wing/wing boundary whose trace is indicated by the [1-100] vector). The directions of the Burgers vectors of dislocations $A$ and $B$ are arrowed.

The geometry of the half-loop dislocations in figs. 2 and 3 suggests a migration of these dislocations along the stripe at some point during growth and annealing, i.e. from top to bottom in the case of the two dislocations in fig. 2. Strong evidence for this is the frequent interaction of these dislocations with threading segments. Indeed, both of the dislocations in fig. 2 are seen to be partially pinned by a threading dislocation.

In order to examine the mechanisms by which the boundary and half-loop dislocations were introduced, the distribution of strain in the films was examined by large angle convergent beam electron diffraction (LACBED). Fig. 4 shows an example of a LACBED pattern taken from a region spanning a wing/wing boundary. The diffraction disc covers a rocking angle of about $6^{\circ}$ (corresponding to the angle of convergence of the incident probe) together with a region in real space several microns across. The trace of the wing/wing boundary is indicated (horizontal arrows). The continuity of the $\{1-100\}$ diffraction lines which run perpendicularly across the boundary signifies a rotation of less than $0.1^{\circ}$ about the boundary normal. In contrast, there is a tilt of about $0.5^{\circ}$ about the [1-100] axis in the boundary plane, which is best revealed by the displacement of the obliquely inclined contours arrowed.

The results in fig. 4 were fairly representative, with measurements from different areas giving tilts across both the wing/wing and seed/wing boundaries of typically $0.3-0.5^{\circ}$ about the [1-100] axis. In images this was often apparent as a change in diffraction contrast across these boundaries. It was found that the tilt was such that the wings usually tilted towards the substrate. Rotations about the boundary normal were found to be smaller than the tilts, typically up to $0.1^{\circ}$, and to vary in sign from one region to another. 


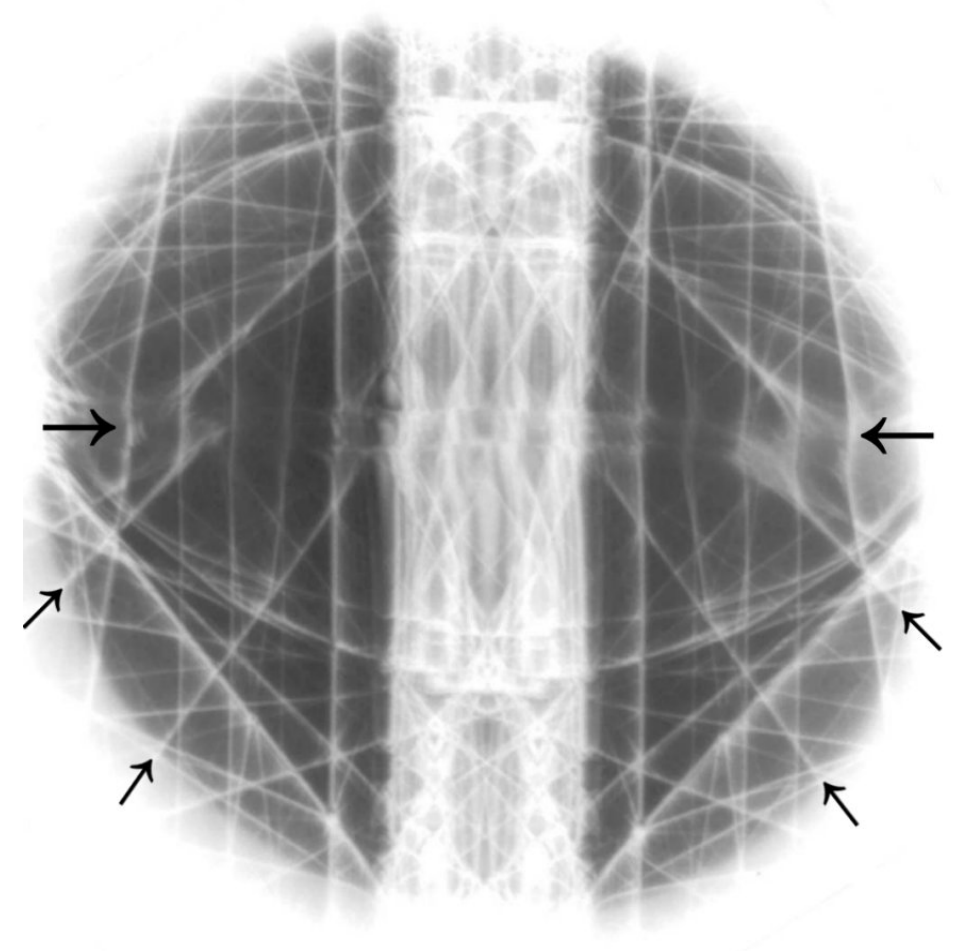

Fig. 4. LACBED pattern showing the crystal rotation across a wing/wing boundary. The horizontal arrows show the trace of the boundary. A tilt across the boundary of about $0.5^{\circ}$ is revealed by displacements of the obliquely inclined contours which are arrowed.

\section{Discussion}

The LACBED results confirm that the arrays of a-type pure edge dislocations that make up the wing/wing and seed/wing boundaries should be described as grain boundary dislocations forming tilt boundaries. The spacing of these dislocations was measured and found to be consistent with the tilt angles recorded. In contrast, the Burgers vectors of the half-loop dislocations correspond partly to tilts (i.e. the component perpendicular to the boundary) and partly to twists about the boundary normal (the component in the boundary plane). The density and irregular spacing of these dislocations along the wings is consistent with the small but varying twists recorded. It is worth noting that the two dislocations $\mathrm{A}$ in fig. 3, if they move in opposite directions as implied by the geometry, introduce twists of opposite sign across this particular boundary.

The apparent migration of the half-loop dislocations, as well as the twist introduced by their movement, suggests that the driving force for their introduction is shear stress acting along the stripe direction. These shear stresses may be introduced during cool-down after growth by the thermal mismatch between $\mathrm{GaN}$ and $\mathrm{SiO}_{2}$, along with uneven yielding at the interface between them. The observation of occasional voids at the $\mathrm{GaN} / \mathrm{SiO}_{2}$ interface is in agreement with this proposal. We propose that the shear stress causes threading a-type dislocations to bend over to generate a segment in the basal plane (fig. 5). Only those a-type dislocations with Burgers vectors $1 / 3$ [1-210] and 1/3[2-1-10] will be affected by this stress, with the boundary dislocations, whose Burgers vectors are 1/3[11-20] being unaffected. 


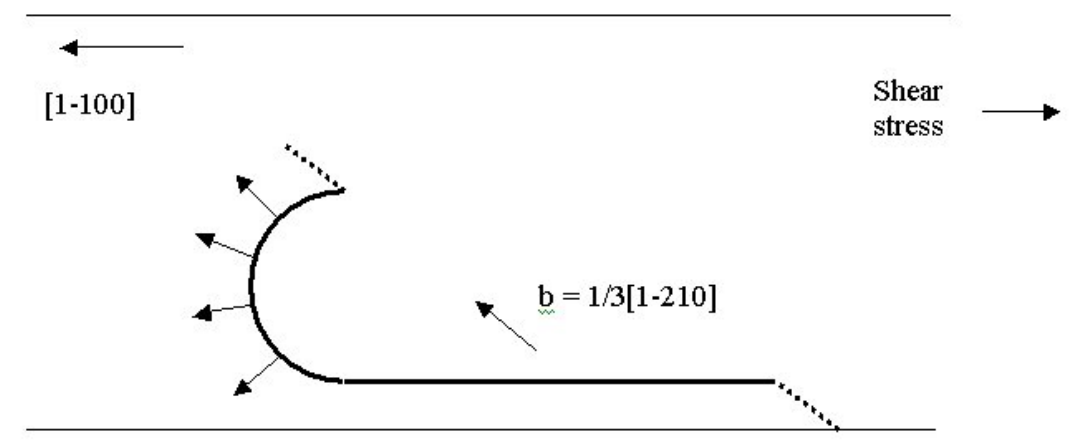

Fig. 5. Schematic view of a wing region (delineated by horizontal lines) in which a half-loop is expanding under the influence of a shear stress acting along the stripe direction. Dotted and bold lines indicate the threading and basal plane segments respectively.

Once the process is initiated, the Peach-Koehler force, given by $\mathrm{F}=\sigma \mathrm{b}$ where $\sigma$ is the stress, acts perpendicular to the dislocation line direction. This will lead to the basal plane segment extending by glide into a half-loop. The threading segments, which can only climb, will then trail the basal plane segment as is apparent from the observations.

The generation of the half-loops is thus seen as irregular process, which depends on nonuniform yielding at the $\mathrm{GaN} / \mathrm{SiO}_{2}$ interface. It is therefore suggested that elimination of these dislocations requires control of bonding and slip at this interface. This might be achieved through surface preparation of the $\mathrm{SiO}_{2}$, or by control of the cool-down cycle following growth.

\section{Summary}

We have shown that two types of a-dislocation are introduced in the wing regions of ELOG samples to describe crystal rotations generated during growth, or as a result of cool-down after growth. Boundary dislocations with edge Burgers vectors are formed at seed/wing and wing/wing boundaries to describe wing tilts of about $0.5^{\circ}$. Half-loops with Burgers vectors at $30^{\circ}$ to the stripe direction describe variable twists generated by shear stresses acting in the stripe direction. They originate from threading dislocations and grow by both glide and climb.

\section{References}

1. T.S. Zheleva, O.H. Lam, M.D. Bremser and R.F. Davis, Appl. Phys. Lett. 71 (1997) 2472.

2. Y. Chen et al., Appl. Phys. Lett. 75 (1999) 2062.

3. A. Sakai, H. Sunakawa and A. Utsui, Appl. Phys. Lett. 73 (1998) 481.

4. T.S. Zheleva et al., Appl. Phys. Lett. 74 (1999) 2492.

\section{Acknowledgements}

ZLW is grateful for support from the U.S. Department of Energy under grant DE-AC0376FS00098 and the U.K. Engineering and Physical Sciences Research Council under grant GR/N16426. 
